CAHIERS DE

NARRATOLOGIE

\section{Cahiers de Narratologie}

Analyse et théorie narratives

32 | 2017

Récit et argumentation, interactions, lieux et dispositifs sociaux

\title{
Les fonctions de la focalisation et du point de vue dans la dynamique de l'intrigue
}

\section{Raphaël Baroni}

\section{(2) OpenEdition}

\section{Journals}

Electronic version

URL: http://journals.openedition.org/narratologie/7851

DOI: 10.4000/narratologie.7851

ISSN: 1765-307X

Publisher

LIRCES

Electronic reference

Raphaël Baroni, «Les fonctions de la focalisation et du point de vue dans la dynamique de l'intrigue ». Cahiers de Narratologie [Online], 32 | 2017, Online since 21 December 2017, connection on 10

December 2020. URL : http://journals.openedition.org/narratologie/7851 ; DOI : https://doi.org/ 10.4000/narratologie.7851

This text was automatically generated on 10 December 2020 .

Article L.117-1 du Code de la propriété intellectuelle. 


\title{
Les fonctions de la focalisation et $\mathrm{du}$ point de vue dans la dynamique de l'intrigue
}

\author{
Raphaël Baroni
}

\section{AUTHOR'S NOTE}

Cet article reprend un certain nombre d'arguments exposés dans mon dernier ouvrage, Les Rouages de l'intrigue, publié aux éditions Slatkine en 2017. Pour en savoir plus : https:// www.slatkine.com/fr/slatkine-reprints-erudition/70192-

book-07102808-9782051028080.html

1 Il faut reconnaître que la narratologie s'est longtemps montrée réticente à opérer une fusion entre les perspectives formelle et fonctionnelle, ce qui reviendrait à décrire les structures narratives en les reliant aux fonctions discursives qu'elles sont susceptibles de remplir dans l'interaction entre le texte et le lecteur. On peut constater que cette réticence s'observe aux deux extrêmes du spectre théorique qui va du formalisme des structuralistes aux approches rhétoriques. En effet, Genette soutenait qu'il est a priori impossible d'affirmer qu'un élément formel du récit, quel qu'il soit, possède "une fonction précisément assignable» (Genette $2007: 420$ ), ce qui explique que sa typologie ne peut être rattachée que de manière anecdotique ou indirecte à la dynamique de la mise en intrigue. Au pôle opposé, dans la version la plus intransigeante des approches rhétoriques, Meir Sternberg s'est servi de la métaphore du "principe de Protée » pour affirmer que «n'importe quel effet peut être produit par un nombre infini de formes, et que n'importe quelle forme peut produire un nombre infini d'effets » $(2011: 40$, m.t.). Ces points de vue, étonnamment convergents pour des auteurs aux positions généralement considérées comme antagonistes ${ }^{1}$, expliquent en grande partie la difficulté de l'émergence d'une véritable stylistique de la dynamique de l'intrigue dans l'histoire de la narratologie. 
2 Ce constat ne nous condamne cependant nullement à faire l'impasse sur une analyse des relations, au moins potentielles, entre forme et fonction. Il s'agit simplement de garder à l'esprit la dépendance de l'effet que peut accomplir une forme donnée envers un contexte d'usage. En conséquence, il faut souligner le danger de généraliser les pseudoéquivalences du type « forme $\mathrm{X}=$ fonction $\mathrm{Y}$ » tout en ménageant malgré tout une analyse $\mathrm{du}$ type : telle forme a telle affinité avec telle(s) fonction(s), et elle pourrait produire tel effet dans telles circonstances ou encore dans ce texte particulier, on constate que telle forme remplit telle fonction quand elle est utilisée de telle manière. L'analyse des affinités entre forme et fonction restera ainsi dans le domaine du virtuel et de l'indétermination relative, plusieurs fonctions, parfois opposées, pouvant être potentiellement associées à une même forme. Toutefois, l'analyse textuelle tirerait certainement profit d'une réflexion générale sur ces affinités potentielles, dans la mesure où elle contribuerait à attirer l'attention sur différents aspects formels - par exemple les temps verbaux, la focalisation ou l'agencement temporel - dont il faut au moins reconnaître qu'ils peuvent avoir une influence directe sur la dynamique de l'intrigue.

3 Ainsi, il serait absurde de ne pas reconnaître qu'une restriction dans la focalisation peut induire de la curiosité, alors qu'un changement de rythme, par exemple un ralentissement dans une scène, est généralement corrélé à l'intensité du suspense. Quoi qu'il en soit, il faut reconnaître que chaque œuvre est susceptible de faire un usage singulier, et plus ou moins original, de tel ou tel paramètre, dont l'effet dépendra de son interaction avec d'autres composantes du récit. Wayne $C$. Booth soulignait ainsi qu'une « technique donnée ne peut pas être jugée sur son utilité pour le roman ou la fiction, mais seulement en fonction de son efficacité dans telles œuvres particulières, ou telles espèces d'œuvres » (Booth 1961 : 111). L'analyse de la dynamique de l'intrigue ne peut donc être menée à son terme qu'en tenant compte du contexte dans lequel s'insère le dispositif textuel susceptible d'intriguer le lecteur. C'est alors, et alors seulement, que les approches formelle et fonctionnelle peuvent fusionner, la première trouvant sa finalité dans la description des moyens textuels déployés par l'auteur pour produire les effets décrits par la seconde : nouer ou dénouer une intrigue, susciter du suspense, inspirer de la curiosité ou surprendre le lecteur.

4 Quoi qu'il en soit, Sternberg et Genette, en dépit de leur scepticisme, et malgré leurs divergences, reconnaissent implicitement, l'un comme l'autre, l'existence de ces affinités entre formes et fonctions. En effet, en faisant reposer sa typologie des intérêts narratifs sur l'interrelation entre la séquence des événements (histoire) et la séquence du discours ( récit), Sternberg (1978) souligne par exemple que le suspense dépend d'une représentation plus ou moins chronologique de l'action, alors que la curiosité dépend au contraire d'une disjonction plus ou moins marquée entre le développement de l'histoire et la progression du récit, ce qui lie de manière fondamentale la catégorie genettienne de l'ordre à la dynamique narrative. Sternberg ajoute que la curiosité repose sur une lacune visible du récit, qui sera comblée ultérieurement, ce qui nous rapproche également des figures genettiennes de la focalisation externe ou de la paralipse. Quant à Genette, dans Discours $d u$ récit, il mentionne presque systématiquement les différentes fonctions rattachables aux figures qu'il répertorie, même si c'est souvent pour montrer, dans un deuxième temps, comment le roman proustien les pervertit ou en transforme le sens. Il s'agit donc de faire preuve de prudence et d'éviter de simplifier des rapports qui dépendent fortement du contexte ou du cotexte, mais non de se borner au constat d'une 
rupture irréconciliable entre les perspectives formaliste et rhétorique, ou entre l'objectivité descriptive et la subjectivité de l'expérience esthétique.

Il parait évident que pour comprendre les mécanismes textuels qui sont au fondement de la dynamique de l'intrigue, il est impossible de faire l'économie d'une étude de la focalisation, étant donné que ce paramètre conditionne de manière directe l'accès aux informations concernant le monde raconté. C'est d'ailleurs sur ce critère du filtrage de l'information que Genette fonde sa distinction entre les différents modes :

le récit peut fournir au lecteur plus ou moins de détails, et de façon plus ou moins directe, et sembler ainsi (pour reprendre une métaphore spatiale courante et commode, à condition de ne pas la prendre au pied de la lettre) se tenir à plus ou moins grande distance de ce qu'il raconte; il peut aussi choisir de régler l'information narrative qu'il livre, non plus par cette sorte de filtrage uniforme, mais selon les capacités de connaissance de telle ou telle partie prenante de l'histoire (personnage ou groupe de personnages), dont il adoptera ou feindra d'adopter ce que l'on nomme couramment la "vision» ou le "point de vue", semblant alors prendre à l'égard de l'histoire (pour continuer la métaphore spatiale) telle ou telle perspective. (Genette $2007: 164$ )

En ce qui concerne la « distance » - à laquelle on peut rattacher en partie l'opposition entre showing et telling, si souvent commentée par les narratologues anglophones depuis les travaux de Lubbock -, il semble évident que la représentation d'une scène dans laquelle le narrateur s'efface pour mettre au premier plan les événements qu'il raconte sera plus favorable à l'établissement du suspense, alors que la discontinuité ou l'opacité relative de la représentation induite par de trop nombreuses digressions du narrateur renforcera plutôt la curiosité. De manière similaire, il paraît logique de supposer qu'une focalisation interne devrait favoriser plutôt l'immersion dans le point de vue du personnage, et donc l'effet de suspense, alors qu'à l'inverse, une focalisation externe, par la restriction de l'information qu'elle suppose, devrait déboucher plutôt sur un effet de curiosité. Il faut toutefois se garder de simplifier ces rapports entre forme et fonction. Ainsi que le signale Genette, il faut commencer par reconnaître que le filtrage du récit par un point de vue interne n'entraîne pas nécessairement un processus d'identification ou d'empathie :

[L]es subtilités narratives du roman moderne, depuis Flaubert et James, comme le style indirect libre, le monologue intérieur ou la focalisation multiple, exercent plutôt, sur le désir d'adhésion du lecteur, des effets négatifs, et contribuent à brouiller les pistes, à égarer les " évaluations ", à décourager les sympathies et les antipathies. (Genette $2007: 421$ )

7 Suzanne Keen, en s'appuyant sur des études empiriques, dresse un constat similaire. Elle souligne notamment la difficulté d'établir des rapports immédiats entre la création d'un effet d'empathie et des techniques narratives spécifiques :

La confirmation [par des études empiriques] de la plupart des hypothèses concernant les liens entre des techniques narratives spécifiques et l'empathie doit encore être apportée dans la plupart des cas, mais le travail qui a été fait, comme souvent, échoue à confirmer pleinement les lieux communs de la narratologie. (Keen 2006 : 216, m.t.)

8 Keen affirme ainsi que ce serait plutôt la présence d'une menace qui induirait de l'empathie envers le personnage, et non le filtrage de l'information par un point de vue interne, qui ne jouerait qu'un rôle marginal :

Certaines techniques de caractérisation des personnages ont effectivement été testées au niveau de la réponse émotionnelle des lecteurs ou de l'empathie. 
L'implication des personnages dans une situation de suspense engendre des réactions physiologiques chez les lecteurs, même quand ces derniers méprisent la qualité du récit. (Keen $2006: 217$, m.t.)

Quoi qu'il en soit, en liant directement sa théorie de la focalisation aux différents degrés de restriction de l'information narrative, le modèle de Genette apparaît comme particulièrement adapté pour servir de base à une interprétation fonctionnelle du filtrage de la narration. Genette distingue en effet trois modes de focalisation en comparant les savoirs auxquels ont accès les personnages aux informations dispensées par les narrateurs : focalisation interne (le narrateur en sait autant que personnage), focalisation externe (le narrateur en sait moins que le personnage) et focalisation zéro (le narrateur en sait plus que le personnage). Il faut noter que dans les récits où les «narrateurs » ne sont pas personnalisés, et pour autant que l'on postule leur existence, la question de savoir ce que ces narrateurs «savent » repose sur une psychologisation abusive. A priori, le fait que nous ayons ou n'ayons pas accès à une information dépend d'une stratégie auctoriale, et ce n'est que dans certains cas spécifiques qu'une restriction de la focalisation peut être motivée en renvoyant à l'ignorance supposée d'un narrateur incarné dans la fiction. En l'occurrence, les romans épistolaires jouent souvent sur de tels décalages entre différents personnages-narrateurs, ce qui peut d'ailleurs constituer le cœur de l'intrigue. Néanmoins, dans la plupart des cas, le filtrage de l'information renvoie en réalité à un rapport beaucoup plus essentiel qui concerne les informations mises à disposition des lecteurs. Sur cette base, on peut reformuler les trois formes de focalisation décrites par Genette de la manière suivante :

1. Focalisation interne : la narration est focalisée sur un personnage en particulier. Le lecteur et le personnage partagent ainsi un savoir commun, mais aussi une ignorance commune, concernant les événements qui forment la trame de l'histoire. C'est un procédé qui n'est pas incompatible avec la curiosité si le personnage est lui-même confronté à une situation énigmatique. Une telle focalisation peut aussi renforcer le suspense, dans la mesure où elle nous rapproche de la perspective ontologique d'un personnage qui ne peut pas deviner ce que lui réserve le futur.

2. Focalisation externe: dans ce cas, l'information se manifeste surtout par une restriction, le lecteur ne pouvant pas accéder aux pensées d'un ou de plusieurs personnages. Ces personnages agissent sans que le lecteur soit en mesure de saisir leur rôle ou leurs intentions, ce qui induit généralement un sentiment de curiosité. C'est un mode fréquent dans les débuts de récits in medias res: en jetant le lecteur au cœur d'une scène, sans exposition préalable, l'auteur pousse le lecteur à s'interroger sur les personnages nouvellement introduits et sur leurs actions ou intentions. Cependant, il faut noter que les intentions des personnages nous sont souvent révélées implicitement par leurs comportements, leurs discours ou leur appartenance à un réseau de "personnages-types " validé par l'environnement culturel de l'œuvre ${ }^{2}$ (Hamon 1983). Dès lors, la focalisation externe ne devient une figure marquée que dans les cas spécifiques où le récit nous encourage à nous interroger sur ce que dissimule tel ou tel personnage, qui nous est explicitement présenté comme étrange, ambigu ou impénétrable.

3. Focalisation zéro: la focalisation zéro s'apparente à une narration dans laquelle le lecteur a accès à davantage d'informations que certains personnages, ce qui permet par exemple d'anticiper la présence d'un danger que le protagoniste ignore. Ce procédé, sur lequel Hitchcock a beaucoup insisté, a amené certains narratologues à souligner l'importance de ce régime de focalisation pour la dynamique du suspense. Marie-Laure Ryan affirme par exemple que « $[\mathrm{d}] \mathrm{e}$ manière à faciliter l'anticipation qui crée le suspense, le texte peut permettre au lecteur d'en savoir plus que le personnage - par exemple en décrivant un autre 
personnage qui tend un piège au héros pendant que ce dernier est absent de la scène " (2001 : 143, m.t.).

10 L'interprétation fonctionnelle du modèle de Genette, bien que facilitée par l'importance qu'il accorde aux capacités de connaissance de telle ou telle partie prenante de l'histoire, révèle cependant certains problèmes, qu'il a lui-même repérés en considérant ce qu'il appelle des « altérations du mode » $(2007: 200-204)$ :

Le type classique de la paralipse, rappelons-le, c'est, dans le code de la focalisation interne, l'omission de telle action ou pensée du héros focal, que ni le héros, ni le narrateur ne peuvent ignorer, mais que le narrateur choisit de dissimuler au lecteur. (Genette $2007: 201$ )

11 Ainsi, Genette reconnaît que ce qu'il définit comme un régime de focalisation « interne » ne correspond pas toujours à un accès complet aux savoirs du personnage focalisé. On trouve de nombreux exemples de telles restrictions, qui sont parfois étendues sur une très vaste échelle. Ainsi, dans la première moitié du roman de Michel Butor La Modification, l'intrigue repose en grande partie sur une énigme, qui consiste à savoir pourquoi le protagoniste se rend à Rome. Bien que l'information narrative soit entièrement filtrée par les pensées du protagoniste, les informations nécessaires à la compréhension de la situation narrative ne sont distillées qu'au gré des souvenirs ou des pensées, qui surgissent à travers un jeu d'associations libres, et qui sont en réalité contraintes par la dynamique très réticente de l'intrigue forgée par l'auteur. Mais cette restriction de l'information n'est pas une "dissonance locale», ou une "infraction isolée » qui s'opposerait au «mode dominant» (Genette 2007 : 201), puisque le narrateur n'est pas conscient de son rôle et ne se trouve donc pas dans une situation de communication habituelle, ce qui pourrait le contraindre à passer par une phase d'exposition ${ }^{3}$. Cet exemple souligne combien il est important de distinguer le point de vue à travers lequel l'information narrative est filtrée de la question de la focalisation, qui ne concerne que le décalage épistémique entre les personnages et les lecteurs.

Dans le sillage de Mieke $\mathrm{Bal}^{4}$, mais en adoptant une perspective plus linguistique, Alain Rabatel (1998) a fait évoluer la compréhension de la construction textuelle du point de vue. Abandonnant le modèle de la triple focalisation, il a insisté sur le fait que les seuls focalisateurs identifiables dans le récit ne peuvent être que le narrateur ou le personnage, et que l'identification de la perspective narrative dépend du repérage d'indices textuels indiquant un débrayage énonciatif, c'est-à-dire le passage occasionnel de la perspective de l'instance qui raconte à celle du personnage. Le récit se trouverait donc ancré par défaut dans la perspective du narrateur, mais par endroits, un débrayage survient lorsqu'un discours est rapporté sur un mode direct ou indirect. Par ailleurs, le cas le plus intéressant survient lorsqu'un débrayage partiel permet de réancrer la perspective dans le point de vue d'un personnage, sans pour autant lui céder directement ou explicitement la parole. Ces figures recoupent en partie ce que d'autres chercheurs ont désigné comme une forme de "psycho-récit» (Cohn 1981) ou comme une figure relevant du discours indirect libre (Banfield 1997 ; Hamburger 1986 ; Patron 2009). Sans entrer dans le détail de cette analyse, nous pouvons cependant noter que, dans un récit verbal, ce sont essentiellement les énoncés situés à l'arrière-plan ${ }^{5}$, et renvoyant aux paroles, aux perceptions ou aux pensées des personnages, qui peuvent manifester un tel débrayage du point de vue :

Ce sont [...] essentiellement les marques et les indices de l'aspectualisation du focalisé qui déterminent l'existence du point de vue et qui nous indiquent à quel focalisateur coréfère la référenciation du focalisé: ces indices et marques 
apparaissent dans les seconds plans des phrases narratives, autour de l'expression

de perceptions intriquées à des pensées. (Rabatel 1998 : 9) audiovisuelles, puisqu'il inclut non seulement les autres sens des personnages, mais également leurs pensées. Toutefois, les remarques de Jost sur la distinction entre focalisation et point de vue demeurent opérantes : la détermination d'un foyer perceptif ou cognitif dans lequel s'ancre le récit ne doit pas être confondu avec la question de la gestion des informations qui permettent de comprendre une situation narrative. C'est ce qui amène d'ailleurs Rabatel à affirmer lui aussi que les restrictions de l'information narrative «tiennent non pas à d'illusoires distinctions de foyer, mais à des stratégies différentes de gestion de l'information narrative, en fonction des intentions communicationnelles de l'écrivain» (1997: 107). Ainsi que l'affirme Burkhard Niederhoff :

Il y a de la place pour les deux [concepts] parce que chacun met en évidence un aspect différent d'un phénomène complexe et difficile à saisir. Le point de vue semble être la métaphore la plus efficace pour les récits qui tentent de rendre l'expérience subjective d'un personnage. Affirmer qu'une histoire est racontée du point de vue d'un personnage a plus de sens que d'affirmer qu'il y a une focalisation interne sur ce personnage. La focalisation est un terme plus approprié lorsqu'on analyse la sélection des informations narratives qui ne servent pas à restituer l'expérience subjective d'un personnage mais à créer d'autres effets, tels que le suspense, le mystère, la perplexité, etc. Pour que la théorie de la focalisation puisse progresser, la conscience des différences entre les deux termes, mais aussi la conscience de leurs forces et de leurs faiblesses respectives, est indispensable. (Niederhoff 2011: \$18, m.t.)

Il ne faudrait donc pas confondre une stratégie communicationnelle, qui peut consister à dissimuler ou à révéler une information cruciale concernant un personnage, avec une stratégie représentationnelle, qui consiste à adopter un point de vue singulier vis-à-vis du monde racontét. La combinaison entre une focalisation "externe » et un point de vue « interne » ne serait donc pas une contradiction ou un paradoxe, mais correspondrait simplement à un cas particulier où un décalage épistémique se manifesterait alors même 
que nous pensions être en symbiose avec le personnage, ce qui produit un effet de proximité étrange. À l'inverse, une focalisation "zéro" liée à un point de vue interne correspondrait à une forme de fusion ironique, à l'instar de cette phrase tirée de Madame Bovary, exemple typique de style indirect libre dont le débrayage énonciatif nous ancre dans le point de vue de Léon, mais dont les italiques et la formulation excessive laissent entendre une autre voix, dans laquelle perce l'ironie d'un auteur implicite: «D'ailleurs, n'était-ce pas une femme du monde, et une femme mariée! une vraie maîtresse enfin?» (Flaubert 1972: 342). On peut aussi imaginer un cas beaucoup plus banal: celui où le lecteur sait des choses que le personnage ignore, tout simplement parce qu'une information lui a été communiquée avant que le récit adopte un point de vue interne.

On voit bien cependant ce qu'il y a de gênant dans ces formulations, qui définissent la même situation narrative avec des termes opposés. Pour éviter de confondre la focalisation avec le point de vue, il faudrait alors probablement renoncer aux termes « internes » et « externes », pour privilégier une typologie plus explicite :

18 1. Le récit focalisé (anciennement focalisation interne) est un récit centré sur un (groupe de) personnage(s). Un tel récit joue sur un partage maximal des connaissances entre le (ou les) personnage(s) et le lecteur. Ce régime de focalisation ne correspond pas nécessairement à un récit adoptant un point de vue interne (ce qui correspondrait à un filtrage du monde raconté par des perceptions ou des pensées subjectives), mais il facilite néanmoins grandement les processus d'identification et confère généralement $\mathrm{au}(\mathrm{x})$ personnage(s) focalisé(s) le statut de protagoniste(s). Ce mode peut engendrer aussi bien des effets de curiosité ou de suspense, dans la mesure où les diagnostics et les pronostics formulés par les personnages sur lesquels le récit est focalisé se confondent généralement avec les hypothèses interprétatives des lecteurs.

2. Le récit à focalisation restreinte (anciennement focalisation externe) se définit par une situation, qui peut être parfois très locale, dans laquelle le lecteur est confronté à un déficit d'information par rapport à un (groupe de) personnage(s), ce qui induit par défaut un effet de curiosité.

20 3. Le récit à focalisation élargie (anciennement focalisation zéro, récit parfois improprement qualifié de narration "omnisciente») divulgue certaines informations auxquelles les personnages n'ont pas accès, ce qui permet souvent de renforcer le suspense d'une scène en anticipant un danger qui menace le(s) protagoniste(s).

On pourrait également faire l'hypothèse de l'existence d'un quatrième pôle, qui correspondrait à un récit qui ne serait pas focalisé, et qui se distinguerait de la focalisation élargie par le fait que l'excès d'information ne pourrait pas être comparé au savoir d'un personnage focal en particulier, mais simplement au fait que, par définition, un narrateur en sait généralement plus que chaque personnage pris individuellement. Je pense néanmoins qu'un tel récit ne correspond pas au régime ordinaire du récit mimétique tel que j'ai défini ce dernier ${ }^{7}$, lequel repose, ainsi que l'affirme Monika Fludernik (1996), sur un ancrage de la représentation dans une expériencialité simulée, ce qui induit naturellement un récit plus ou moins focalisé sur un ou plusieurs personnages qui incarnent cette expérience. Le récit non focalisé pourrait se rattacher en revanche au pôle du récit informatif, par exemple au genre de l'historiographie, car dans ce cas, le narrateur poursuit un idéal de neutralité et d'objectivité, ce qui l'amène à éviter de se focaliser sur un protagoniste en particulier au détriment d'autres personnages, le but 
étant par ailleurs d'offrir une représentation aussi transparente que possible d'un événement en donnant accès à un maximum de sources d'information.

Philippe Carrard (2013), à travers une étude détaillée des modalités d'écriture des historiens français contemporains, a d'ailleurs montré que ces derniers adoptent généralement un mode de narration en surplomb, offrant une sorte de cartographie historique des événements, ce qui exclut les modes de focalisations interne et externe (dans ma typologie: focalisation sur un personnage ou focalisation restreinte), qu'il considère comme des procédés plutôt mobilisés par les fictions. Il parle alors de focalisation zéro, mais dans le modèle que je propose, cette dernière ne peut être définie en tant que focalisation élargie, dans la mesure où l'excès de savoir manifesté par l'historien ne peut être comparé à un foyer de connaissance spécifique, qui renverrait à un personnage focal. Certes, on peut comparer le savoir des historiens aux celui des acteurs de l'histoire, ce qui conduit à affirmer par exemple que le recul permet de mieux comprendre les événements, ou au contraire, que la distance temporelle rend certaines informations inaccessibles (par exemple à travers la disparition de sources ou l'accès impossible aux intentions de tel ou tel agent). Quoi qu'il en soit, une telle comparaison n'a pas grandchose à voir avec les variations de focalisation des récits mimétiques, qui visent surtout à entretenir la dynamique de l'intrigue en entretenant la curiosité ou le suspense d'une scène. Il serait donc plus correct de parler, dans ce cas, d'un récit non focalisé, qui constitue une quatrième option, surtout actualisée dans des formes narratives adoptant un registre non mimétique (ou informatif), c'est-à-dire dans des récits qui ne cherchent pas à être immersifs, qui renoncent à ancrer la représentation dans le monde raconté. À l'inverse, à partir du moment où il $\mathrm{y}$ a ce que les linguistes cognitivistes définissent comme un " déplacement déictique » (Duchan et al. 1995 ; Philippe 2000), c'est-à-dire un réancrage de la narration dans ce que le linguiste allemand Karl Bühler (1934) a appelé une deixis am phantasma, on peut faire l'hypothèse que le récit - qui privilégie par ailleurs la scène au sommaire-, se trouve nécessairement en régime de focalisation sur un personnage, même si cette dernière peut faire l'objet d'altérations locales, dont les figures sont la restriction ou l'élargissement.

On comprend aussi pourquoi, d'un côté, on aboutit naturellement à un modèle binaire, alors que de l'autre, nous sommes confrontés à un système à trois ou quatre pôles. Comme l'affirme Rabatel, un point de vue ne peut être qu'interne ou externe, mais la focalisation, en reposant sur un étalonnage de l'information narrative comparée au savoir que possède un personnage focal, aboutit naturellement à envisager deux autres alternatives: une restriction ou un élargissement. L'alternative que constitue le récit non focalisé renvoie simplement au cas où un tel étalonnage de l'information n'aurait pas de pertinence directe.

\section{Point de vue interne / externe \\ Focalisation \\ foc. restreinte $<$ foc. sur le savoir d'un personnage $<$ foc. élargie alternative : récit non focalisé}

En se basant sur cette double reformulation de la triade genettienne, il devient beaucoup moins paradoxal d'affirmer que, dans un récit qui adopte globalement le point de vue interne d'un personnage, on peut malgré tout se trouver dans un régime de focalisation restreinte, comme dans La Modification. Par ailleurs, ainsi que je l'ai déjà mentionné, dans 
le cinéma d'épouvante, il est fréquent que le récit adopte le point de vue visuel du psychopathe ou du prédateur (effet d'ocularisation interne dans la typologie de Jost) alors que le régime de focalisation doit être interprété comme élargi par rapport à la perspective de la victime potentielle. Certes, nous partageons un savoir avec le requin des Dents de la mer concernant la localisation de sa proie, mais ce que nous voyons à travers ses yeux, c'est surtout une baigneuse ignorant un danger imminent. Dans ce cas, c'est bien l'ignorance de la baigneuse qui est le vrai moteur du suspense, et non le savoir partagé avec le requin. Cette situation, par son caractère stéréotypé, souligne l'efficacité d'une analyse qui découple focalisation et point de vue ${ }^{8}$.

Il faut ajouter que lorsque nous sommes confrontés à une interaction entre plusieurs personnages, si le récit se focalise sur l'un d'entre eux, les autres se trouveront par défaut dans un régime de focalisation restreinte, du moins si ces derniers paraissent dissimuler quelque chose au personnage focal. Il est ensuite possible, en jouant sur ce que Genette définirait comme un jeu de focalisations variables, de passer d'une perspective à l'autre, de manière à dénouer les mystères. Cette variation se manifeste souvent par une segmentation de la narration, par exemple par des changements de chapitres, dont la cohérence interne repose très souvent sur le choix d'un personnage focal.

En conclusion, nous constatons que, bien que la construction textuelle du point de vue constitue un élément central dans la dynamique de l'intrigue, il est impossible d'associer de manière univoque un effet déterminé à un point de vue donné. En revanche, on remarque qu'il y a de fortes affinités entre certains régimes de focalisation et les effets de curiosité (foc. restreinte) et de suspense (foc. élargie). En d'autres termes, l'approche linguistique de Rabatel peut nous permettre de définir assez précisément le point de vue adopté par la narration à tel endroit du récit, mais la corrélation entre l'analyse linguistique d'un débrayage énonciatif et les effets de curiosité ou de suspense ne peut être établie qu'en recourant à l'analyse conjointe des régimes de focalisation, qui sont déterminés à partir de contextes narratifs et discursifs déterminés. Ce qui importe pour la dynamique de l'intrigue, c'est le degré de transparence des personnages et la compréhension relative des situations dans lesquelles ils sont engagés, les variations de point de vue n'étant que l'un des paramètres déterminant ces facteurs. Il faut ajouter que la détermination du personnage focal, que ce soit à l'échelle d'un roman ou d'une scène, repose sur un processus interprétatif qui peut être assez complexe, et vis-à-vis duquel aucun outil linguistique ne permet de trancher de manière indiscutable. Malgré ce constat quelque peu équivoque, il n'en demeure pas moins que l'analyse du point de vue et de la focalisation constituera presque toujours un passage obligé pour l'analyse de la tension narrative, puisque cette dernière dépend étroitement de la modalisation d'un savoir tout au long de la progression du lecteur dans le récit.

\section{BIBLIOGRAPHY}

Bal, Mieke (1977), Narratologie, Essais sur la signification narrative dans quatre romans modernes, Paris, Klincksieck. 
Banfield, Ann (1997), Phrases sans paroles. Théorie du récit et du style indirect libre, Paris, Seuil.

Baroni, Raphaël (2017), Les Rouages de l'intrigue. Nouveaux outils de la narratologie postclasssique pour l'analyse des textes littéraires, Genève, Slatkine érudition.

Booth, Wayne C. (1977 [1961]), « Distance et point de vue. Essai de classification », in Poétique du récit, R. Barthes et al. (dir.), Paris, Seuil, coll. « Points », p. 85-113.

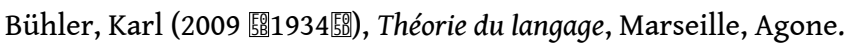

Carrard, Philippe (2013), Le Passé mis en texte. Poétique de l'historiographie français contemporaine, Paris, Armand Colin.

Cohn, Dorrit (1981), La Transparence intérieure, Paris, Seuil.

Duchan, Judith F., Gail A. Bruder \& Lynne E. Hewitt (1995), Deixis in Narrative : A Cognitive Science Perspective, Hillsdale, Lawrence Erlbaum Associates.

Dufays, Jean-Louis (2010), Stéréotype et lecture. Essai sur la réception littéraire, Bruxelles, Peter Lang, coll. « Théocrit ».

Flaubert, Gustave (1972), Madame Bovary, Paris, Gallimard, coll. « Folio ».

Fludernik, Monika (1996), Towards a « Natural » Narratology, London, Routledge.

Genette, Gérard (2007), Discours du récit, Paris, Seuil, coll. « Points ».

Hamburger, Käte (1986), Logique des genres littéraires, Paris, Seuil.

Hamon, Philippe (1983), Le personnel du roman. Le système des personnages dans les Rougon-Macquart d'Émile Zola, Genève, Droz.

Jost, François, (1989 [1987]), L'CEil-caméra. Entre film et roman, Lyon, PUL.

Keen, Suzanne (2006), « A Theory of Narrative Empathy », Narrative, n¹4, p. 207-236.

Niederhoff, Burkhard (2011), « Focalization », The Living Handbook of Narratology. Consulté le 19 mai 2017. URL : http://www.lhn.uni-hamburg.de/article/focalization

Patron, Sylvie (2009), Le Narrateur, Paris, Armand Colin.

Philippe, Gilles (2000), « L'ancrage énonciatif des récits de fiction », Langue française, n 128 p. 3-8.

Rabatel, Alain (1998), La construction textuelle du point de vue, Paris, Delachaux et Niestlé.

Rabatel, Alain (1997), «L'introuvable focalisation externe. De la subordination de la vision externe au point de vue du personnage ou au point du vue du narrateur », Littérature, $n^{\circ} 107, \mathrm{p}$. 88-113.

Schaeffer, Jean-Marie (1999), Pourquoi la fiction?, Paris, Seuil.

Sternberg, Meir (2011), « Reconceptualizing Narratology. Arguments for a Functionalist and Constructivist Approach to Narrative », Enthymema, n4, p. 35-50.

Sternberg, Meir (1993 [1978]), Expositional modes and Temporal ordering in Fiction, Bloomington, Indiana University Press.

Weinrich, Harald (1973), Le Temps, Paris, Seuil. 


\section{NOTES}

1. Sternberg va jusqu'à décrire Genette comme son "plus grand ennemi ", pas en "termes personnels », précise-t-il, mais en termes «théoriques » (2011:40, m.t.).

2. De ce fait leur action potentiellement stéréotypée devient prévisible (avec effet inverse dans des utilisations à contre-emploi). Je remercie Marc Marti de m'avoir fait remarquer l'importance de cet aspect, qui relève de la problématique générale de la stéréotypie, qui joue évidemment un rôle central dans la lecture (Dufays 2010).

3. Ainsi que l'explique Booth, les «narrateurs conscients d'eux-mêmes en tant qu'écrivains» s'opposent à ceux « qui semblent ne pas savoir qu'ils écrivent, pensent, parlent ou "filtrent" une œuvre littéraire » (Booth 1961 : 99).

4. Mieke Bal (1977) est la première à avoir critiqué la typologie des focalisations de Genette en distinguant les pôles du focalisateur et du focalisé.

5. Pour une définition de la notion d'arrière-plan et son lien avec l'usage de l'imparfait (en contraste avec le passé simple ou le passé composé), voir de Weinrich (1973).

6. Sur la notion " posture immersive ", je renvoie aux travaux de Schaeffer (1999).

7. Voir Baroni (2017 : 25-36).

8. Pour une application littéraire, voir Baroni (2017 : 149-156).

\section{ABSTRACTS}

Narratology has long been reluctant to link descriptive analysis of narrative structures with the interpretation of their discursive functions. Yet, starting from a dynamic conception of plot - which emphasizes its function to arouse the reader's interest and direct his or her attention towards a possible outcome -, we will show that it is relatively easy to associate different forms of focalization with effects such as suspense and curiosity. Nevertheless, such an interpretation leads to a clear distinction between the textual construction of the point of view, and focalization as defined by Genette. In addition, in order to avoid confusing point of view and focalization, we propose to adopt a new terminology for the latter. If the point of view can only be internal (i.e. anchored in the subjectivity of a character) or external, focalization, on the contrary, may be marked by a restriction, an equality, or an excess when compared with the knowledge of a focal character. The possibility of unfocalized narratives will also be considered, a case that should not be confused with a simple excess of knowledge in a narrative focalized on the fate of a character.

On fera d'abord le constat que la narratologie a longtemps rechigné à lier l'analyse descriptive des structures narratives à l'interprétation de leurs fonctions discursives. En partant d'une conception dynamique de l'intrigue, qui met au premier plan sa fonction, qui est de susciter l'intérêt du lecteur et d'orienter son attention en direction d'un éventuel dénouement, nous montrerons qu'il est pourtant relativement facile d'associer différents régimes de focalisation à des effets tels que le suspense ou la curiosité. Néanmoins, une telle interprétation conduit à distinguer clairement la construction textuelle du point de vue de la focalisation telle que Genette l'a définie. Par ailleurs, de manière à éviter de confondre point de vue et focalisation, nous proposons d'adopter une nouvelle terminologie pour cette dernière. Si le point de vue ne 
peut être qu'interne (c'est-à-dire ancré dans la subjectivité d'un personnage) ou externe, la focalisation, au contraire, peut-être marquée par une restriction, une égalité ou un élargissement quand on la compare avec le savoir d'un personnage focal. On évoquera également l'éventualité de récits non-focalisés, qui ne se confondent pas avec un excès de savoir dans un récit focalisé sur le destin d'un personnage.

INDEX

Keywords: focalization, point of view, plot, suspense, curiosity

Mots-clés: focalisation, point de vue, intrigue, suspense, curiosité

AUTHOR

RAPHAËL BARONI

Université de Lausanne 